\title{
Some Hermite-Hadamard type inequalities for harmonically extended s-convex functions
}

\section{Chun-Long Lij ${ }^{\mathrm{a}}$, Shan-He Wub,*}

${ }^{a}$ College of Mathematics, Inner Mongolia University for Nationalities, Tongliao City, Inner Mongolia Autonomous Region, 028043, China. ${ }^{b}$ Department of Mathematics, Longyan University, Longyan, Fujian 364012, China.

\author{
Communicated by Y. Hu
}

\begin{abstract}
In this paper, we establish some inequalities of Hermite-Hadamard type for functions whose derivatives absolute values are harmonically extended s-convex functions. (C)2017 All rights reserved.

Keywords: Harmonically extended s-convex function, Hermite-Hadamard type inequalities, integral inequalities. 2010 MSC: 26A51, 26D15, 41A55.
\end{abstract}

\section{Introduction}

The following definitions are well-known in the literature.

Definition 1.1. A function $f: I \subseteq \mathbb{R}=(-\infty,+\infty) \rightarrow \mathbb{R}$ is said to be convex function if

$$
f(t x+(1-t) y) \leqslant t f(x)+(1-t) f(y)
$$

holds for all $x, y \in I$ and $t \in[0,1]$.

In $[1,4]$ the concept of s-convex functions below was given.

Definition $1.2([1,4])$. Let $s \in(0,1]$ be a real number. A function $f: \mathbb{R} \rightarrow \mathbb{R}_{0}=[0, \infty)$ is said to be $s$-convex (in the second sense) if

$$
f(t x+(1-t) y) \leqslant t^{s} f(x)+(1-t)^{s} f(y)
$$

holds for all $x, y \in I$ and $t \in[0,1]$.

In [16] the definition of extended s-convex functions was put forward, i.e.,

Definition 1.3 ([16]). A function $f: I \subseteq \mathbb{R} \rightarrow \mathbb{R}$ is said to be extended s-convex if

$$
f(t x+(1-t) y) \leqslant t^{s} f(x)+(1-t)^{s} f(y)
$$

holds for all $x, y \in I$ and $t \in(0,1)$ and for some fixed $s \in[-1,1]$.

\footnotetext{
*Corresponding author

Email addresses: lichunlong70@163.com (Chun-Long Li), shanhewu@163.com (Shan-He Wu)
} 
Definition 1.4 ([6]). Let $I \subseteq \mathbb{R} \backslash\{0\}$ be a real interval. A function $f: I \rightarrow \mathbb{R}$ is said to be harmonically convex, if

$$
f\left(\frac{x y}{t x+(1-t) y}\right) \leqslant t f(y)+(1-t) f(x)
$$

for all $x, y \in I$ and $t \in[0,1]$. If the inequality in (1.1) is reversed, then $f$ is said to be harmonically concave.

A formal definition for harmonically s-convex functions is stated as follows (see $[7,9])$ :

Definition $1.5([7,9])$. A function $f: I \subseteq \mathbb{R}_{+}=(0,+\infty) \rightarrow \mathbb{R}$ is said to be harmonically s-convex function of second kind, where $s \in(0,1]$, if

$$
f\left(\frac{x y}{t x+(1-t) y}\right) \leqslant t^{s} f(y)+(1-t)^{s} f(x), \quad x, y \in I, \quad t \in[0,1] .
$$

Next, let us recall the concepts of harmonically P-convex and harmonically s-Godunova-Levin convex functions.

Definition 1.6 ([9]). A function $f: I \subseteq \mathbb{R}_{+} \rightarrow \mathbb{R}$ is said to be harmonically P-convex function, if

$$
f\left(\frac{x y}{t x+(1-t) y}\right) \leqslant f(x)+f(y), \quad x, y \in I, \quad t \in[0,1]
$$

Definition 1.7 ([9]). A function $f: I \subseteq \mathbb{R}_{+} \rightarrow \mathbb{R}$ is said to be harmonically Godunova-Levin convex function, if

$$
f\left(\frac{x y}{t x+(1-t) y}\right) \leqslant \frac{f(y)}{t}+\frac{f(x)}{1-t^{\prime}}, \quad x, y \in I, \quad t \in(0,1) .
$$

Definition 1.8 ([9]). A function $f: I \subseteq \mathbb{R}_{+} \rightarrow \mathbb{R}$ is said to be harmonically s-Godunova-Levin convex function of second kind, if

$$
f\left(\frac{x y}{t x+(1-t) y}\right) \leqslant \frac{f(y)}{t^{s}}+\frac{f(x)}{(1-t)^{s}}, \quad x, y \in I, \quad t \in(0,1), \quad s \in(0,1] .
$$

In order to unify the above concepts, we focus on the definition of harmonically extended s-convex functions below.

Definition 1.9 ([9]). A function $f: I \subseteq \mathbb{R}_{+} \rightarrow \mathbb{R}$ is said to be harmonically extended s-convex, if

$$
f\left(\frac{x y}{t x+(1-t) y}\right) \leqslant t^{s} f(y)+(1-t)^{s} f(x), \quad x, y \in I, \quad t \in(0,1), \quad s \in[-1,1] .
$$

It is obvious that the harmonically extended 0-convex function and harmonically extended -1-convex function are just the harmonically P-convex functions (Definition 1.6) and harmonically Godunova-Levin convex functions (Definition 1.7), respectively.

In $[3,8,10]$ and $[5]$, the following Hermite-Hadamard type inequalities for functions whose derivatives absolute values are convex (or s-convex) functions were established.

Theorem 1.10 ([3]). Let $\mathrm{f}: \mathrm{I}^{\circ} \subseteq \mathbb{R} \rightarrow \mathbb{R}$ be a differentiable mapping on $\mathrm{I}^{\circ}, \mathrm{a}, \mathrm{b} \in \mathrm{I}^{\circ}$ with $\mathrm{a}<\mathrm{b}$. If $\left|\mathrm{f}^{\prime}\right|$ is convex on $[\mathrm{a}, \mathrm{b}]$, then the following inequality holds:

$$
\left|\frac{f(a)+f(b)}{2}-\frac{1}{b-a} \int_{a}^{b} f(x) d x\right| \leqslant \frac{(b-a)\left(\left|f^{\prime}(a)\right|+\left|f^{\prime}(b)\right|\right)}{8} .
$$

Theorem 1.11 ([10]). Let $\mathrm{f}: \mathrm{I} \subseteq \mathbb{R} \rightarrow \mathbb{R}$ be differentiable on $\mathrm{I}^{\circ}, \mathrm{a}, \mathrm{b} \in \mathrm{I}$ with $\mathrm{a}<\mathrm{b}$. If $\left|\mathrm{f}^{\prime}\right|^{\mathrm{q}}$ is convex on $[\mathrm{a}, \mathrm{b}]$ and $\mathrm{q} \geqslant 1$, then

$$
\left|\frac{f(a)+f(b)}{2}-\frac{1}{b-a} \int_{a}^{b} f(x) d x\right| \leqslant \frac{b-a}{4}\left(\frac{\left|f^{\prime}(a)\right|^{q}+\left|f^{\prime}(b)\right|^{q}}{2}\right)^{1 / q},
$$

and

$$
\left|f\left(\frac{a+b}{2}\right)-\frac{1}{b-a} \int_{a}^{b} f(x) d x\right| \leqslant \frac{b-a}{4}\left(\frac{\left|f^{\prime}(a)\right|^{q}+\left|f^{\prime}(b)\right|^{q}}{2}\right)^{1 / q} .
$$


Theorem 1.12 ([8]). Let $\mathrm{f}: \mathrm{I} \subseteq \mathbb{R}_{0} \rightarrow \mathbb{R}$ be differentiable on $\mathrm{I}^{\circ}, \mathrm{a}, \mathrm{b} \in \mathrm{I}$ with $\mathrm{a}<\mathrm{b}$. If $\left|\mathrm{f}^{\prime}\right|^{\mathrm{q}}$ is s-convex on $[\mathrm{a}, \mathrm{b}]$ for some fixed $\mathrm{s} \in(0,1]$ and $\mathrm{q} \geqslant 1$, then

$$
\left|\frac{f(a)+f(b)}{2}-\frac{1}{b-a} \int_{a}^{b} f(x) d x\right| \leqslant \frac{b-a}{2}\left(\frac{1}{2}\right)^{1-1 / q}\left[\frac{2+1 / 2^{s}}{(s+1)(s+2)}\right]^{1 / q}\left[\left|f^{\prime}(a)\right|^{q}+\left|f^{\prime}(b)\right|^{q}\right]^{1 / q} .
$$

Theorem 1.13 ([5]). Let $\mathrm{f}: \mathrm{I} \subseteq \mathbb{R}_{0} \rightarrow \mathbb{R}$ be differentiable on $\mathrm{I}^{\circ}, \mathrm{a}, \mathrm{b} \in \mathrm{I}$ with $\mathrm{a}<\mathrm{b}$, and $\mathrm{f}^{\prime} \in \mathrm{L}[\mathrm{a}, \mathrm{b}]$. If $\left|\mathrm{f}^{\prime}\right|^{\mathrm{q}}$ is $\mathrm{s}$-convex on $[\mathrm{a}, \mathrm{b}]$ for some fixed $\mathrm{s} \in(0,1]$ and $\mathrm{q}>1$, then

$$
\begin{aligned}
& \left|f\left(\frac{a+b}{2}\right)-\frac{1}{b-a} \int_{a}^{b} f(x) d x\right| \leqslant \frac{b-a}{4}\left[\frac{1}{(s+1)(s+2)}\right]^{1 / q}\left(\frac{1}{2}\right)^{1 / p} \\
& \quad \times\left\{\left[\left|f^{\prime}(a)\right|^{q}+(s+1)\left|f^{\prime}\left(\frac{a+b}{2}\right)\right|^{q}\right]^{1 / q}+\left[\left|f^{\prime}(b)\right|^{q}+(s+1)\left|f^{\prime}\left(\frac{a+b}{2}\right)\right|^{q}\right]^{1 / q}\right\},
\end{aligned}
$$

where $\frac{1}{\mathrm{p}}+\frac{1}{\mathrm{q}}=1$.

In recent years, some other kinds of Hermite-Hadamard type inequalities were generated in, for example, [2, 11-15] and [17].

In this paper we shall establish some new Hermite-Hadamard type inequalities for harmonically extended s-convex functions.

\section{Two lemmas}

To establish some new Hermite-Hadamard type inequalities for harmonically extended s-convex functions, we need the following integral identities:

Lemma 2.1. Let $f: I \subseteq \mathbb{R}_{+} \rightarrow \mathbb{R}$ be a twice differentiable function on $\mathrm{I}^{\circ}, \mathrm{a}, \mathrm{b} \in \mathrm{I}^{\circ}$ with $\mathrm{a}<\mathrm{b}$. If $\mathrm{f}^{\prime \prime} \in \mathrm{L}_{1}([\mathrm{a}, \mathrm{b}])$, then

$$
\begin{aligned}
& \frac{f(a)+f(b)}{2}+\frac{a b\left[f^{\prime}(b)-f^{\prime}(a)\right]}{2(b-a)}-\frac{1}{b-a} \int_{a}^{b} f(x) d x \\
& \quad=\frac{1}{2(a b)^{2}} \int_{0}^{1}\left[(1-t) a^{2}+t b^{2}\right]\left(\frac{t}{a}+\frac{1-t}{b}\right)^{-4} f^{\prime \prime}\left(\left(\frac{t}{a}+\frac{1-t}{b}\right)^{-1}\right) d t .
\end{aligned}
$$

Proof. Letting $x=\left(\frac{t}{a}+\frac{1-t}{b}\right)^{-1}$ for $t \in[0,1]$, then

$$
\begin{aligned}
\frac{1}{2(a b)^{2}} \int_{0}^{1}\left[(1-t) a^{2}+t b^{2}\right]\left(\frac{t}{a}+\frac{1-t}{b}\right)^{-4} f^{\prime \prime}\left(\left(\frac{t}{a}+\frac{1-t}{b}\right)^{-1}\right) d t \\
\quad=\frac{1}{2(b-a)^{2}} \int_{a}^{b}[a(x-a) x+b(b-x) x] f^{\prime \prime}(x) d x \\
=\frac{a b\left[f^{\prime}(b)-f^{\prime}(a)\right]}{2(b-a)}-\frac{1}{2(b-a)} \int_{a}^{b}[a(2 x-a)+b(b-2 x)] f^{\prime}(x) d x \\
=\frac{f(a)+f(b)}{2}+\frac{a b\left[f^{\prime}(b)-f^{\prime}(a)\right]}{2(b-a)}-\frac{1}{b-a} \int_{a}^{b} f(x) d x .
\end{aligned}
$$

Lemma 2.1 is proved.

Lemma 2.2. Let $\mathrm{f}: \mathrm{I} \subseteq \mathbb{R}_{+} \rightarrow \mathbb{R}$ be a twice differentiable function on $\mathrm{I}^{\circ}, \mathrm{a}, \mathrm{b} \in \mathrm{I}^{\circ}$ with $\mathrm{a}<\mathrm{b}$. If $\mathrm{f}^{\prime \prime} \in \mathrm{L}_{1}([\mathrm{a}, \mathrm{b}])$, then

$$
\frac{\frac{1}{a b}\left[\frac{1}{a^{2}} f(a)+\frac{1}{b^{2}} f(b)\right]}{2}-\frac{1}{b-a} \int_{a}^{b}\left(\frac{3}{x^{4}}-\frac{2}{H(a, b) x^{3}}\right) f(x) d x
$$




$$
=\frac{(b-a)^{2}}{2(a b)^{3}} \int_{0}^{1} t(1-t)\left(\frac{t}{a}+\frac{1-t}{b}\right)^{-2} f^{\prime \prime}\left(\left(\frac{t}{a}+\frac{1-t}{b}\right)^{-1}\right) d t,
$$

where $\mathrm{H}(\mathrm{a}, \mathrm{b})=\frac{2 \mathrm{ab}}{\mathrm{a}+\mathrm{b}}$.

Proof. Letting $x=\left(\frac{t}{a}+\frac{1-t}{b}\right)^{-1}$ for $t \in[0,1]$, then

$$
\begin{aligned}
& \frac{(b-a)^{2}}{2(a b)^{3}} \int_{0}^{1} t(1-t)\left(\frac{t}{a}+\frac{1-t}{b}\right)^{-2} f^{\prime \prime}\left(\left(\frac{t}{a}+\frac{1-t}{b}\right)^{-1}\right) d t \\
& \quad=\frac{1}{2(b-a)} \int_{a}^{b}\left(\frac{1}{x}-\frac{1}{b}\right)\left(\frac{1}{a}-\frac{1}{x}\right) f^{\prime \prime}(x) d x \\
& =-\frac{1}{2(b-a)} \int_{a}^{b}\left[\frac{2}{x^{3}}-\frac{1}{x^{2}}\left(\frac{1}{a}+\frac{1}{b}\right)\right] f^{\prime}(x) d x \\
& =\frac{\frac{1}{a b}\left[\frac{1}{a^{2}} f(a)+\frac{1}{b^{2}} f(b)\right]}{2}+\frac{1}{2(b-a)} \int_{a}^{b}\left[-\frac{6}{x^{4}}+\frac{2}{x^{3}}\left(\frac{1}{a}+\frac{1}{b}\right)\right] f(x) d x \\
& =\frac{\frac{1}{a b}\left[\frac{1}{a^{2}} f(a)+\frac{1}{b^{2}} f(b)\right]}{2}-\frac{1}{b-a} \int_{a}^{b}\left(\frac{3}{x^{4}}-\frac{2}{H(a, b) x^{3}}\right) f(x) d x .
\end{aligned}
$$

The proof of Lemma 2.2 is completed.

\section{Main results}

Our main results are given in the following theorems.

Theorem 3.1. Let $\mathrm{f}: \mathrm{I} \subseteq \mathbb{R}_{+} \rightarrow \mathbb{R}$ be a twice differentiable function on $\mathrm{I}^{\circ}, \mathrm{a}, \mathrm{b} \in \mathrm{I}$ with $\mathrm{a}<\mathrm{b}$, and $\mathrm{f}^{\prime \prime} \in$ $\mathrm{L}_{1}([\mathrm{a}, \mathrm{b}])$. If $\left|\mathrm{f}^{\prime \prime}\right|^{\mathrm{q}}$ for $\mathrm{q} \geqslant 1$ is harmonically extended $\mathrm{s}$-convex on $\mathrm{I}^{\circ}$ for some fixed $\mathrm{s} \in(-1,1]$, then

$$
\begin{aligned}
\mid \frac{f(a)+f(b)}{2} & +\frac{a b\left[f^{\prime}(b)-f^{\prime}(a)\right]}{2(b-a)}-\frac{1}{b-a} \int_{a}^{b} f(x) d x \mid \\
& \leqslant \frac{[H(a, b, 0)]^{1-1 / q}}{2(a b)^{2}}\left[H(a, b, s)\left|f^{\prime \prime}(a)\right|^{q}+H(b, a, s)\left|f^{\prime \prime}(b)\right|^{q}\right]^{1 / q}
\end{aligned}
$$

where

$$
H(a, b, s)=\frac{(s+1)\left[a^{2}+(s+2) b^{2}\right] a^{4}+\left[2 a^{2}+(s+1) b^{2}\right] b^{4}}{(s+3)(s+2)(s+1)}
$$

Proof. Using Lemma 2.1, and the Hölder's integral inequality and the harmonically extended s-convexity of function $\left|f^{\prime \prime}\right| q$, we obtain

$$
\begin{aligned}
\mid \frac{f(a)+f(b)}{2}+ & \frac{a b\left[f^{\prime}(b)-f^{\prime}(a)\right]}{2(b-a)}-\frac{1}{b-a} \int_{a}^{b} f(x) d x \mid \\
\leqslant & \frac{1}{2(a b)^{2}} \int_{0}^{1}\left[(1-t) a^{2}+t b^{2}\right]\left(\frac{t}{a}+\frac{1-t}{b}\right)^{-4}\left|f^{\prime \prime}\left(\left(\frac{t}{a}+\frac{1-t}{b}\right)^{-1}\right)\right| d t \\
\leqslant & \frac{1}{2(a b)^{2}}\left[\int_{0}^{1}\left[(1-t) a^{2}+t b^{2}\right]\left(\frac{t}{a}+\frac{1-t}{b}\right)^{-4} d t\right]^{1-1 / q} \\
& \times\left[\int_{0}^{1}\left[(1-t) a^{2}+t b^{2}\right]\left(\frac{t}{a}+\frac{1-t}{b}\right)^{-4}\left|f^{\prime \prime}\left(\left(\frac{t}{a}+\frac{1-t}{b}\right)^{-1}\right)\right|^{q} d t\right]^{1 / q}
\end{aligned}
$$




$$
\begin{aligned}
\leqslant & \frac{1}{2(a b)^{2}}\left[\int_{0}^{1}\left[(1-t) a^{2}+t b^{2}\right]\left(\frac{t}{a}+\frac{1-t}{b}\right)^{-4} d t\right]^{1-1 / q} \\
& \times\left[\int_{0}^{1}\left[(1-t) a^{2}+t b^{2}\right]\left(\frac{t}{a}+\frac{1-t}{b}\right)^{-4}\left[t^{s}\left|f^{\prime \prime}(a)\right|^{q}+(1-t)^{s}\left|f^{\prime \prime}(b)\right|^{q}\right] d t\right]^{1 / q} .
\end{aligned}
$$

Since $\left(t a^{-1}+(1-t) b^{-1}\right)^{-p} \leqslant t a^{p}+(1-t) b^{p}$ for $t \in[0,1]$ and $p>1$, we have used the facts

$$
\begin{aligned}
\int_{0}^{1}[(1 & \left.-t) a^{2}+t b^{2}\right]\left(\frac{t}{a}+\frac{1-t}{b}\right)^{-4} d t \\
\leqslant & \int_{0}^{1}\left[(1-t) a^{2}+t b^{2}\right]\left[t a^{4}+(1-t) b^{4}\right] d t=H(a, b, 0) \\
& \quad \times \int_{0}^{1} t^{s}\left[(1-t) a^{2}+t b^{2}\right]\left(\frac{t}{a}+\frac{1-t}{b}\right)^{-4} d t \\
\leqslant & \int_{0}^{1} t^{s}\left[(1-t) a^{2}+t b^{2}\right]\left[t a^{4}+(1-t) b^{4}\right] d t=H(a, b, s), \\
& \quad \times \int_{0}^{1}(1-t)^{s}\left[(1-t) a^{2}+t b^{2}\right]\left(\frac{t}{a}+\frac{1-t}{b}\right)^{-4} d t \\
\leqslant & \int_{0}^{1}(1-t)^{s}\left[(1-t) a^{2}+t b^{2}\right]\left[t a^{4}+(1-t) b^{4}\right] d t=H(b, a, s) .
\end{aligned}
$$

The proof of Theorem 3.1 is completed.

Corollary 3.2. Under the conditions of Theorem 3.1, when $\mathrm{q}=1$, then

$$
\begin{aligned}
\mid \frac{f(a)+f(b)}{2} & +\frac{a b\left[f^{\prime}(b)-f^{\prime}(a)\right]}{2(b-a)}-\frac{1}{b-a} \int_{a}^{b} f(x) d x \mid \\
& \leqslant \frac{1}{2(a b)^{2}}\left[H(a, b, s)\left|f^{\prime \prime}(a)\right|+H(b, a, s)\left|f^{\prime \prime}(b)\right|\right] .
\end{aligned}
$$

Theorem 3.3. Let $\mathrm{f}: \mathrm{I} \subseteq \mathbb{R}_{+} \rightarrow \mathbb{R}$ be a twice differentiable function on $\mathrm{I}^{\circ}, \mathrm{a}, \mathrm{b} \in \mathrm{I}$ with $\mathrm{a}<\mathrm{b}$, and $\mathrm{f}^{\prime \prime} \in$ $\mathrm{L}_{1}([\mathrm{a}, \mathrm{b}])$. If $\left|\mathrm{f}^{\prime \prime}\right|^{\mathrm{q}}$ for $\mathrm{q} \geqslant 1$ is harmonically extended s-convex on $\mathrm{I}$ for $\mathrm{s}=-1$, then

$$
\begin{aligned}
& \left|\frac{\frac{1}{a b}\left[\frac{1}{a^{2}} f(a)+\frac{1}{b^{2}} f(b)\right]}{2}-\frac{1}{b-a} \int_{a}^{b} f(x) d x\right| \\
& \leqslant \frac{b-a}{2(a b)^{2} L(a, b)}\left[\frac{a b[(a+b)-2 L(a, b)]}{b-a}\right]^{1-1 / q} \\
& \quad \times\left[b[L(a, b)-a]\left|f^{\prime \prime}(a)\right|^{q}+a[b-L(a, b)]\left|f^{\prime \prime}(b)\right|^{q}\right]^{1 / q},
\end{aligned}
$$

where $\mathrm{L}(\mathrm{u}, \boldsymbol{v})$ is the logarithmic mean defined by

$$
L(u, v)= \begin{cases}\frac{v-u}{\ln v-\ln u}, & u \neq v, \\ u, & u=v, v>0 .\end{cases}
$$

Proof. Since $\left|f^{\prime \prime}\right|^{q}$ is harmonically extended -1-convex function on $I^{\circ}$, by Lemma 2.2 and the Hölder's integral inequality, we have

$$
\left|\frac{\frac{1}{a b}\left[\frac{1}{a^{2}} f(a)+\frac{1}{b^{2}} f(b)\right]}{2}-\frac{1}{b-a} \int_{a}^{b} f(x) d x\right|
$$




$$
\begin{aligned}
\leqslant & \frac{(b-a)^{2}}{2(a b)^{3}}\left[\int_{0}^{1} t(1-t)\left(\frac{t}{a}+\frac{1-t}{b}\right)^{-2} d t\right]^{1-1 / q} \\
& \times\left[\int_{0}^{1} t(1-t)\left(\frac{t}{a}+\frac{1-t}{b}\right)^{-2}\left|f^{\prime \prime}\left(\left(\frac{t}{a}+\frac{1-t}{b}\right)^{-1}\right)\right| q d\right]^{1 / q} \\
\leqslant & \frac{(b-a)^{2}}{2(a b)^{3}}\left[\int_{0}^{1} t(1-t)\left(\frac{t}{a}+\frac{1-t}{b}\right)^{-2} d t\right]^{1-1 / q} \\
& \times\left[\int_{0}^{1} t(1-t)\left(\frac{t}{a}+\frac{1-t}{b}\right)^{-2}\left[t^{-1}\left|f^{\prime \prime}(a)\right|^{q}+(1-t)^{-1}\left|f^{\prime \prime}(b)\right|^{q}\right] d t\right]^{1 / q} \\
= & \frac{b-a}{2(a b)^{2} L(a, b)}\left[\frac{a b[(a+b)-2 L(a, b)]}{b-a}\right]^{1-1 / q} \\
& \times\left[b[L(a, b)-a]\left|f^{\prime \prime}(a)\right|^{q}+a[b-L(a, b)]\left|f^{\prime \prime}(b)\right|^{q}\right]^{1 / q} .
\end{aligned}
$$

The proof of Theorem 3.3 is completed.

Corollary 3.4. Under the conditions of Theorem 3.3, when $\mathrm{q}=1$, then

$$
\begin{aligned}
\mid \frac{\frac{1}{a b}\left[\frac{1}{a^{2}} f(a)+\frac{1}{b^{2}} f(b)\right]}{2} & -\frac{1}{b-a} \int_{a}^{b} f(x) d x \mid \\
& \leqslant \frac{(b-a)^{2}}{2(a b)^{2}}\left[\frac{b[1-a L(a, b)]}{b-a}\left|f^{\prime \prime}(a)\right|+\frac{a[b L(a, b)-1]}{b-a}\left|f^{\prime \prime}(b)\right|\right] .
\end{aligned}
$$

Theorem 3.5. Let $\mathrm{f}: \mathrm{I} \subseteq \mathbb{R}_{+} \rightarrow \mathbb{R}$ be a twice differentiable function on $\mathrm{I}^{\circ}, \mathrm{a}, \mathrm{b} \in \mathrm{I}$ with $\mathrm{a}<\mathrm{b}$, and $\mathrm{f}^{\prime \prime} \in$ $\mathrm{L}_{1}([\mathrm{a}, \mathrm{b}])$. If $\left|\mathrm{f}^{\prime \prime}\right|^{\mathrm{q}}$ is harmonically extended s-convex on $\mathrm{I}$ for some fixed $\mathrm{s} \in(-1,1]$ and $\mathrm{q}>1$, then

$$
\begin{aligned}
\mid \frac{f(a)+f(b)}{2}+ & \frac{a b\left[f^{\prime}(b)-f^{\prime}(a)\right]}{2(b-a)}-\frac{1}{b-a} \int_{a}^{b} f(x) d x \mid \leqslant \frac{1}{2(a b)^{2 / q}} \\
& \times\left[\frac{(q-1)\left(b^{\frac{2(q+1)}{q-1}}(a+b(q-1)+3 a q)-a^{\frac{2(q+1)}{q-1}}(b+a(q-1)+3 b q)\right)}{2(b-a)(3 q+1)(q+1)}\right]^{1-1 / q} \\
& \times\left[\frac{a^{2}+(s+1) b^{2}}{(s+2)(s+1)}\left|f^{\prime \prime}(a)\right|^{q}+\frac{(s+1) a^{2}+b^{2}}{(s+2)(s+1)}\left|f^{\prime \prime}(b)\right|^{q}\right]^{1 / q} .
\end{aligned}
$$

Proof. By Lemma 2.1, and the Hölder's integral inequality and the harmonically extended s-convexity of function $\left|f^{\prime \prime}\right| q$, we deduce

$$
\begin{aligned}
\mid \frac{f(a)+f(b)}{2}+ & \frac{a b\left[f^{\prime}(b)-f^{\prime}(a)\right]}{2(b-a)}-\frac{1}{b-a} \int_{a}^{b} f(x) d x \mid \\
\leqslant & \frac{1}{2(a b)^{2}}\left[\int_{0}^{1}\left[(1-t) a^{2}+t b^{2}\right]\left(\frac{t}{a}+\frac{1-t}{b}\right)^{-4 q /(q-1)} d t\right]^{1-1 / q} \\
& \times\left[\int_{0}^{1}\left[(1-t) a^{2}+t b^{2}\right]\left|f^{\prime \prime}\left(\left(\frac{t}{a}+\frac{1-t}{b}\right)^{-1}\right)\right| d t\right]^{1 / q} \\
\leqslant & \frac{1}{2(a b)^{2}}\left[\int_{0}^{1}\left[(1-t) a^{2}+t b^{2}\right]\left(\frac{t}{a}+\frac{1-t}{b}\right)^{-4 q /(q-1)} d t\right]^{1-1 / q}
\end{aligned}
$$




$$
\begin{aligned}
& \times\left[\int_{0}^{1}\left[(1-t) a^{2}+t b^{2}\right]\left[t^{s}\left|f^{\prime \prime}(a)\right|^{q}+(1-t)^{s}\left|f^{\prime \prime}(b)\right|^{q}\right] d t\right]^{1 / q} \\
\leqslant & \frac{1}{2(a b)^{2 / q}}\left[\frac{(q-1)\left[b^{\frac{2(q+1)}{q-1}}(a+b(q-1)+3 a q)-a^{\frac{2(q+1)}{q-1}}(b+a(q-1)+3 b q)\right]}{2(b-a)(3 q+1)(q+1)}\right]^{1-1 / q} \\
& \times\left[\frac{a^{2}+(s+1) b^{2}}{(s+2)(s+1)}\left|f^{\prime \prime}(a)\right|^{q}+\frac{(s+1) a^{2}+b^{2}}{(s+2)(s+1)}\left|f^{\prime \prime}(b)\right|^{q}\right]^{1 / q} .
\end{aligned}
$$

This completes the proof of Theorem 3.5 .

Theorem 3.6. Let $\mathrm{f}: \mathrm{I} \subseteq \mathbb{R}_{+} \rightarrow \mathbb{R}$ be a twice differentiable function on $\mathrm{I}^{\circ}, \mathrm{a}, \mathrm{b} \in \mathrm{I}$ with $\mathrm{a}<\mathrm{b}$, and $\mathrm{f}^{\prime \prime} \in$ $\mathrm{L}_{1}([\mathrm{a}, \mathrm{b}])$. If $\left|\mathrm{f}^{\prime \prime}\right|^{\mathrm{q}}$ is harmonically extended -1-convex on I for $\mathrm{q}>1$, then

$$
\begin{aligned}
\mid \frac{\frac{1}{a b}\left[\frac{1}{a^{2}} f(a)+\frac{1}{b^{2}} f(b)\right]}{2} & -\frac{1}{b-a} \int_{a}^{b} f(x) d x \mid \\
& \leqslant \frac{(b-a)^{2}}{4(a b)^{3}}\left[\frac{a^{2 q /(q-1)}+b^{2 q /(q-1)}}{6}\right]^{1-1 / q}\left[\left|f^{\prime \prime}(a)\right|^{q}+\left|f^{\prime \prime}(b)\right|^{q}\right]^{1 / q} .
\end{aligned}
$$

Proof. By Lemma 2.2, and the Hölder's integral inequality and the harmonically extended -1-convexity of function $\left|f^{\prime \prime}\right| q$, we deduce

$$
\begin{aligned}
\mid \frac{\frac{1}{a b}\left[\frac{1}{a^{2}} f(a)+\frac{1}{b^{2}} f(b)\right]}{2} & -\frac{1}{b-a} \int_{a}^{b} f(x) d x \mid \\
\leqslant & \frac{(b-a)^{2}}{2(a b)^{3}} \int_{0}^{1} t(1-t)\left(\frac{t}{a}+\frac{1-t}{b}\right)^{-2}\left|f^{\prime \prime}\left(\left(\frac{t}{a}+\frac{1-t}{b}\right)^{-1}\right)\right| d t \\
\leqslant & \frac{(b-a)^{2}}{2(a b)^{3}}\left[\int_{0}^{1} t(1-t)\left(\frac{t}{a}+\frac{1-t}{b}\right)^{-2 q /(q-1)} d t\right]^{1-1 / q} \\
& \times\left[\int_{0}^{1} t(1-t)\left[t^{-1}\left|f^{\prime \prime}(a)\right|^{q}+(1-t)^{-1}\left|f^{\prime \prime}(b)\right|^{q}\right] d t\right]^{1 / q} \\
\leqslant & \frac{(b-a)^{2}}{4(a b)^{3}}\left[\frac{a^{2 q /(q-1)}+b^{2 q /(q-1)}}{6}\right]^{1-1 / q}\left[\left|f^{\prime \prime}(a)\right|^{q}+\left|f^{\prime \prime}(b)\right|^{q}\right]^{1 / q} .
\end{aligned}
$$

Theorem 3.6 is proved.

Theorem 3.7. Let $\mathrm{f}: \mathrm{I} \subseteq \mathbb{R}_{+} \rightarrow \mathbb{R}$ be a twice differentiable function on $\mathrm{I}^{\circ}, \mathrm{a}, \mathrm{b} \in \mathrm{I}$ with $\mathrm{a}<\mathrm{b}$, and $\mathrm{f}^{\prime \prime} \in$ $\mathrm{L}_{1}([\mathrm{a}, \mathrm{b}])$. If $\left|\mathrm{f}^{\prime \prime}\right|^{\mathrm{q}}$ for $\mathrm{q}>1$ is harmonically extended s-convex on I for some fixed $\mathrm{s} \in(-1,1]$, then

$$
\begin{aligned}
\mid \frac{f(a)+f(b)}{2}+\frac{a b\left[f^{\prime}(b)-f^{\prime}(a)\right]}{2(b-a)} & -\frac{1}{b-a} \int_{a}^{b} f(x) d x \mid \\
\leqslant & \frac{1}{2(a b)^{2}}\left\{\frac{q-1}{(2 q-1)(3 q-2)\left(b^{2}-a^{2}\right)^{2}}\right. \\
& \times\left[b^{\frac{2(2 q-1)}{q-1}}\left(b^{\frac{2(3 q-1)}{q-1}}(q-1)+a^{\frac{4 q}{q-1}} b^{2}(2 q-1)-a^{\frac{2(3 q-1)}{q-1}}(3 q-2)\right)\right. \\
& \left.\left.+a^{\frac{2(2 q-1)}{q-1}}\left(a^{\frac{2(3 q-1)}{q-1}}(q-1)+a^{2} b^{\frac{4 q}{q-1}}(2 q-1)-b^{\frac{2(3 q-1)}{q-1}}(3 q-2)\right)\right]\right\}^{1-1 / q} \\
& \times\left[\frac{\left|f^{\prime \prime}(a)\right|^{q}+\left|f^{\prime \prime}(b)\right|^{q}}{s+1}\right]^{1 / q} .
\end{aligned}
$$


Proof. From Lemma 2.1, and the Hölder's integral inequality and the harmonically extended s-convexity of function $\left|f^{\prime \prime}\right| q$, we obtain

$$
\begin{aligned}
\mid \frac{f(a)+f(b)}{2}+\frac{a b\left[f^{\prime}(b)-f^{\prime}(a)\right]}{2(b-a)} & -\frac{1}{b-a} \int_{a}^{b} f(x) d x \mid \\
\leqslant & \frac{1}{2(a b)^{2}}\left[\int_{0}^{1}\left[(1-t) a^{2}+t b^{2}\right]^{q /(q-1)}\left(\frac{t}{a}+\frac{1-t}{b}\right)^{-4 q /(q-1)} d t\right]^{1-1 / q} \\
& \times\left[\int_{0}^{1}\left|f^{\prime \prime}\left(\left(\frac{t}{a}+\frac{1-t}{b}\right)^{-1}\right)\right|^{q} d t\right]^{1 / q} \\
\leqslant & \frac{1}{2(a b)^{2}}\left[\int_{0}^{1}\left[(1-t) a^{2}+t b^{2}\right]^{q /(q-1)}\left(\frac{t}{a}+\frac{1-t}{b}\right)^{-4 q /(q-1)} d t\right]^{1-1 / q} \\
& \times\left[\int_{0}^{1}\left[t^{s}\left|f^{\prime \prime}(a)\right|^{q}+(1-t)^{s}\left|f^{\prime \prime}(b)\right|^{q}\right] d t\right]^{1 / q} \\
\leqslant & \frac{1}{2(a b)^{2}}\left\{\frac{q q-1}{(2 q-1)(3 q-2)\left(b^{2}-a^{2}\right)^{2}}\right. \\
& \times\left[b^{\frac{2(2 q-1)}{q-1}}\left(b^{\frac{2(3 q-1)}{q-1}}(q-1)+a^{\frac{4 q}{q-1}} b^{2}(2 q-1)-a^{\frac{2(3 q-1)}{q-1}}(3 q-2)\right)\right. \\
& \left.\left.+a^{\frac{2(2 q-1)}{q-1}}\left(a^{\frac{2(3 q-1)}{q-1}}(q-1)+a^{2} b^{\frac{4 q}{q-1}}(2 q-1)-b^{\frac{2(3 q-1)}{q-1}}(3 q-2)\right)\right]\right\}^{1-1 / q} \\
& \times\left[\frac{\left|f^{\prime \prime}(a)\right|^{q}+\left|f^{\prime \prime}(b)\right|^{q}}{s+1 / q}\right]^{1 / q} \cdot
\end{aligned}
$$

This completes the proof of Theorem 3.7.

\section{Acknowledgment}

The work of the first author was supported in part by the National Science Foundation of China (No.11361038). The work of the second author was supported in part by the Natural Science Foundation of Fujian Province of China (No.2016J01023).

\section{References}

[1] W. W. Breckner, Stetigkeitsaussagen für eine Klasse verallgemeinerter konvexer Funktionen in topologischen linearen Räumen, (German) Publ. Inst. Math. (Beograd) (N.S.), 23 (1978), 13-20. 1, 1.2

[2] F.-X. Chen, S.-H. Wu, Some Hermite-Hadamard type inequalities for harmonically s-convex functions, Scientific World J., 2014 (2014), 7 pages. 1

[3] S. S. Dragomir, R. P. Agarwal, Two inequalities for differentiable mappings and applications to special means of real numbers and to trapezoidal formula, Appl. Math. Lett., 11 (1998), 91-95. 1, 1.10

[4] H. Hudzik, L. Maligranda, Some remarks on s-convex functions, Aequationes Math., 48 (1994), 100-111. 1, 1.2

[5] S. Hussain, M. I. Bhatti, M. Iqbal, Hadamard-type inequalities for s-convex functions, I, Punjab Univ. J. Math. (Lahore), 41 (2009), 51-60. 1, 1.13

[6] İ. İşcan, Hermite-Hadamard type inequalities for harmonically convex functions, Hacet. J. Math. Stat., 43 (2014), $935-942$. 1.4

[7] İ. İşcan, S.-H. Wu, Hermite-Hadamard type inequalities for harmonically convex functions via fractional integrals, Appl. Math. Comput., 238 (2014), 237-244. 1, 1.5

[8] U. S. Kirmaci, M. Klaričić Bakula, M. E. Özdemir, J. Pečarić, Hadamard-type inequalities for s-convex functions, Appl. Math. Comput., 193 (2007), 26-35. 1, 1.12

[9] M. A. Noor, K. I. Noor, M. U. Awan, S. Costache, Some integral inequalities for harmonically h-convex functions, Politehn. Univ. Bucharest Sci. Bull. Ser. A Appl. Math. Phys., 77 (2015), 5-16. 1, 1.5, 1.6, 1.7, 1.8, 1.9 
[10] C. E. M. Pearce, J. Pečarić, Inequalities for differentiable mappings with application to special means and quadrature formulæ, Appl. Math. Lett., 13 (2000), 51-55. 1, 1.11

[11] S.-H. Wu, On the weighted generalization of the Hermite-Hadamard inequality and its applications, Rocky Mountain J. Math., 39 (2009), 1741-1749. 1

[12] S.-H. Wu, B. Sroysang, J.-S. Xie, Y.-M. Chu, Parametrized inequality of Hermite-Hadamard type for functions whose third derivative absolute values are quasi-convex, SpringerPlus, 4 (2015), 9 pages.

[13] B.-Y. Xi, R.-F. Bai, F. Qi, Hermite-Hadamard type inequalities for the $\mathrm{m}$ - and $(\alpha, \mathrm{m})$-geometrically convex functions, Aequationes Math., 84 (2012), 261-269.

[14] B.-Y. Xi, F. Qi, Some Hermite-Hadamard type inequalities for differentiable convex functions and applications, Hacet. J. Math. Stat., 42 (2013), 243-257.

[15] B.-Y. Xi, F. Qi, Hermite-Hadamard type inequalities for geometrically r-convex functions, Studia Sci. Math. Hungar., 51 (2014), 530-546. 1

[16] B.-Y. Xi, F. Qi, Inequalities of Hermite-Hadamard type for extended s-convex functions and applications to means, J. Nonlinear Convex Anal., 16 (2015), 873-890. 1, 1.3

[17] B.-Y. Xi, T.-Y. Zhang, F. Qi, Some inequalities of Hermite-Hadamard type for m-harmonic-arithmetically convex functions, ScienceAsia, 41 (2015), 357-361. 1 